\title{
Chromosome X riddle in SARS-CoV-2 (COVID-19) - related lung pathology
}

\author{
Evangelos Tsiambas ${ }^{1,2,3}$ - Aristeidis Chrysovergis ${ }^{4}$ - Vasileios Papanikolaou ${ }^{4} \cdot$ Nicholas Mastronikolis $^{5}$. \\ Vasileios Ragos ${ }^{6}$ - Nikolaos Kavantzas ${ }^{2}$ - Andreas C. Lazaris ${ }^{2}$ - Efstratios Patsouris ${ }^{2}$. Christos Riziotis ${ }^{7}$. \\ Minas Paschopoulos ${ }^{8} \cdot$ Efthymios Kyrodimos $^{4}$
}

Received: 3 June 2020 / Accepted: 13 July 2020 / Published online: 20 July 2020

(C) Arányi Lajos Foundation 2020

\section{Dear Editor,}

Coronaviruses (CoVs) are responsible for three worldwide outbreaks in the last twenty years: Severe Acute Respiratory Syndrome (SARS-CoV) in 2002/2003, Middle-East Respiratory Syndrome (MERS- CoV) in 2012/2013, and the current 2019/2020 Severe Acute Respiratory Syndrome-2 (SARS-CoV-2). Concerning their structural and molecular characteristics, CoVs are members of Nidovirales order which also include Roniviridae and Arteriviridae groups. CoVs family comprises four major sub-classifications: alpha-, beta-, gamma-, and delta-CoVs. The SARS-CoV-2 is responsible for the Coronavirus Disease-19 (COVID-19) pandemic initially reported in Wuhan, Hubei, $\mathrm{Cn}$ - and belongs to lineage $\mathrm{b}$ of beta-CoVs demonstrating a strong phylogenetic similarity with BatCoVRaTG13 type. Concerning its genomic structure, a large non-segmented, positive-sense RNA molecule of approximately $30 \mathrm{~kb}$ has been detected and analyzed in conjunction with the corresponding RNA-dependent RNA-polymerase (Rd-Rp) that is essential for its replication in the cytoplasm

Dr. Tsiambas is an active participant and affiliate of Dept of CME, Harvard Medical School, MA, USA educational activities in Cytology.

For PubMed citation Tsiambas E, Chrysovergis A, Papanikolaou V, Mastronikolis N, Ragos V, Kavantzas N, Lazaris AC, Patsouris E, Riziotis C, Paschopoulos M, Kyrodimos E

Evangelos Tsiambas

tsiambasecyto@yahoo.gr

1 Department of Cytology, VA Hospital (NIMTS), Athens $401 \& 417$, Greece

2 Department of Pathology, Medical School, National and Kapodistrian University of Athens, Athens, Greece

3 Department of CME, Harvard Medical School, Boston, MA, USA

4 1st ENT Department, Hippocration Hospital, National and Kapodistrian, University of Athens, Athens, Greece of the target epithelial cells. Analyzing SARS-CoV-2 spherical virion's structure (diam $\sim 100 \mathrm{~nm}$ ), research groups have confirmed that there are four main proteins including the spike surface glycoprotein $(\mathrm{S})$, the main or matrix protein $(\mathrm{M})$, the envelope protein (E), and finally the nucleocapsid protein (NC), whereas a variety of non-structural proteins have been also identified. S glycoprotein projections -consisting of two subunits S1/S2- provide a unique crown-like formation (corona) on virion's surface. Concerning their functional role, $\mathrm{S} 1$ represents the main receptor-binding domain (RBD), whereas S2 is involved in the virus-cell membrane fusion mechanism interacting with proteases, such as furin, thrypsin, cathepsin or serino-protease TMPRSS2 [1, 2]. Novel molecular and structural/crystallographic analyses have focused on a specific cell membrane receptor - the human angiotensinconverting enzyme 2 (hACE2) - which is the main targetfunctional receptor for SARS-CoV-2 cell attack, attachment and entry that leads to $\mathrm{S} 1$ and $\mathrm{S} 2$ subunits activation. Interestingly, the furin protease provides cleavage of $\mathrm{S}$

5 Department of Otorhinolaryngology, Head and Neck Surgery, Medical School, University of Patras, Patras, Greece

6 Department of Maxillofacial Surgery, School of Health Sciences, University of Ioannina, Ioannina, Greece

7 Theoretical and Physical Chemistry Institute, Photonics for Nanoapplications Laboratory, National Hellenic Research Foundation, Athens, Greece

8 Department of Obstetrics and Gynaecology, School of Health Sciences, University of Ioannina, Ioannina, Greece 
glycoprotein to S1/S2 subunits in lung infected epithelia, dramatically in type II pneumonocytes [3]. Mechanisms of furin interaction with other critical molecules including hypoxia factors seem to be also significant regarding its expression and activity in SARS-CoV-2 cell entry [4].

Rapidly developed bilateral pneumonia is the most severe complication affecting critically the lower respiratory tract anatomy leading also to increased mortality rates, especially in males, older, immunocompromised patients and others with a history of metabolic (diabetes mellitus, obesity) or other chronic diseases (malignancies, cardio-vascular and pulmonary failure). A recently published study - based on autopsy procedures - revealed serious specific pathological findings representative of a distinctive acute interstitial pneumonia in COVID-19 molecularly positive victims. Concerning lung epithelia, infected tissues are characterized by diffuse alveolar damage combined with microvascular lesions and also with intra/extra-vascular fibrin deposition. Intravascular trapping of neutrophils combined with microthrombi in arterioles are also observed, whereas gross pulmonary thromboemboli with pulmonary infarcts and diffuse hemorrhage are also included in the pathological landscape [5]. Lymphocytic pneumonitis, pericarditis and myocarditis were also detected in the corresponding examined tissues of dead patients.

According to the latest published epidemiologic data, SARS-CoV-2-mediated COVID-19 pandemic demonstrates aggressive clinic-pathological profiles in significant subsets of the infected patients - especially in males- and for this reason the role of chromosome X that hosts the hACE2 gene (band Xp22.2) seem to be critical. Genomic analyses in humans have shown that this chromosome contains approximately 800 genes that encode for protein products. It is also well established that males and females retain one of their mother's X chromosomes, whereas females retain another X chromosome from their father. Based on this inheritance pattern, mutations, deletions, amplifications or single nucleotide polymorphisms (SNPs) in X linked genes - such as hACE2 affect directly their expression profiles in males leading to altered proteins, since they have only one $\mathrm{X}$ chromosome hosting one copy of each gene. In contrast, female carriers of abnormal genes demonstrate a normal phenotype due to the second normal and functional gene copy. A prospective cohort study analyzed a dataset including information for incidence rates, infectivity potential and phenotype characteristics in COVID-19 patients in U.S.A. hospitals. They reported that males demonstrated more frequently a progressively aggressive clinical image than females, needing admission to an intensive care unit and oxygen supply for a long time [6]. Furthermore, they reported significantly higher mortality rates among male infected patients compared to females, although the age-dependent mortality risk was equal for both genders.

Another molecular study based on genome-wide linkage scan analysis, explored the role of SNPs in a specific clinicpathological entity -familial intracranial aneurysms- in Japanese families. Among the analyzed genes, hACE2 demonstrated specific SNPs in the majority of the examined cases, whereas none of them was strongly involved in the development and progression of the aneurysms [7]. Similarly, another A/G SNP based analysis in Chinese people showed increased level of $\mathrm{G}$ allele frequencies -especially in male patients with metabolic syndrome - correlated to hypertension [8]. Concerning SARS-CoV-2 cell membrane binding, the impact of these SPNs in potential structural modifications and expression patterns of the hACE2 molecule remains unknown. Interestingly, differences in response to the current viral infection regarding males and females, could be partially explained by the activation of immune regulatory X-linked genes, such as the Toll -like Receptors 7 and 8 (TLR7/8), which lay
Fig. 1 Schematic presentation of $\mathrm{X}$-linked hACE2 and other critical genes in COVID-19. Females host two alleles, whereas males only one. SNPs, rearrangements, epigenetic changes (red spot: abnormal allele, green spot: normal) on Xp22.2 and other Xp bands affect directly normal structure, expression and function of the corresponding proteins in males. (hACE2: human AngiotensinConverting Enzyme 2, TLR7/8: Toll -like Receptors 7 and 8, FOXP3: Forkhead Box P3, RGN: ReGucalciN; SNP: single nucleotide polymorphisms; $\mathrm{Ca}^{2+}$ : calcium)

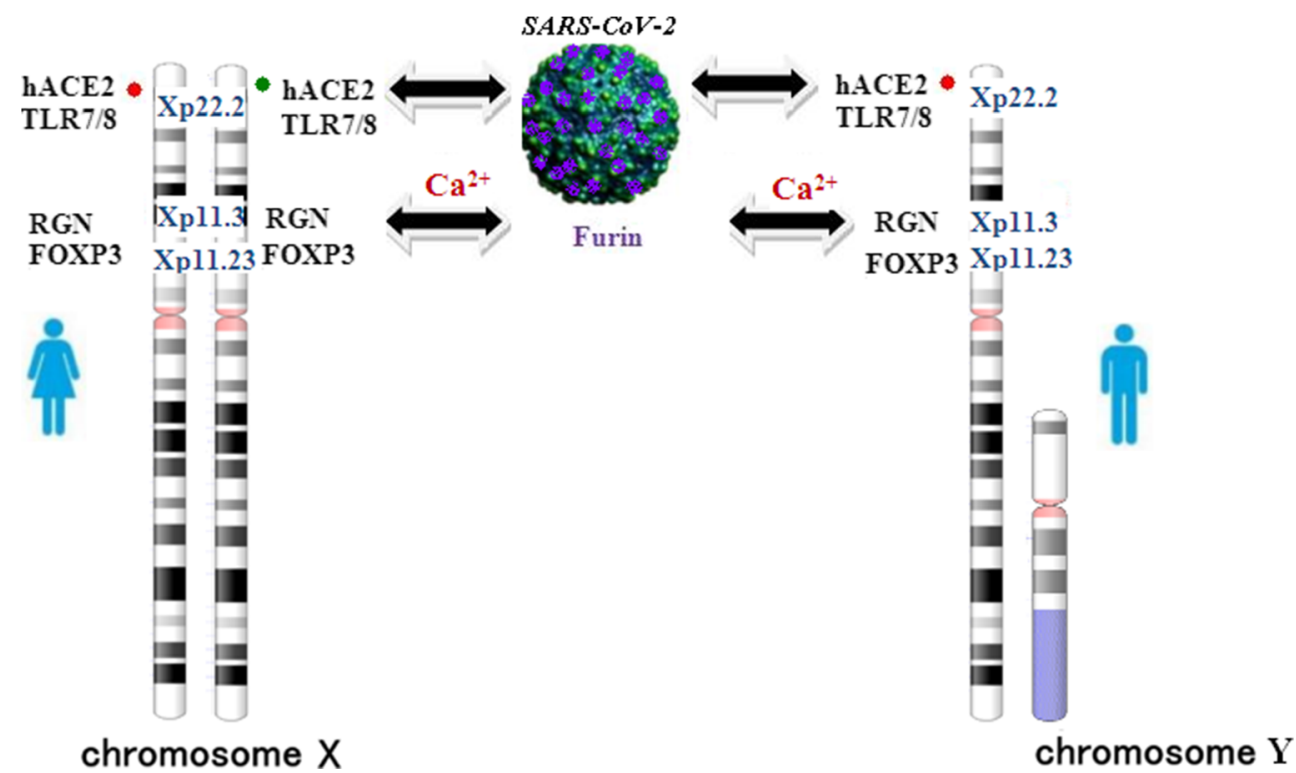


equally to hACE2 gene (band Xp22.2) and also the Forkhead Box P3 (FOXP3) gene located on Xp11.23 [9]. Additionally, hACE2 is found to be significantly overexpressed in cigarette smokers especially in men which represent the major percentage worldwide [10]. Besides SNP modifications in the current chromosome $\mathrm{X}$ region, epigenetic changes including promoter hypomethylation leads to aberrant hACE2 over expression affecting negatively the response to lung severe infection in patients with immune diseases, such as systemic lupus erythematous [11]. Chromosome X hosts another critical gene, the RGN (band Xp11.3), which encodes for regucalcin, an important calcium $\left(\mathrm{Ca}^{2+}\right)$-binding protein involved in intracellular $\mathrm{Ca}^{2+}$ homeostasis principally expressed in hepatocytes. Interestingly, regucalcin is expressed also in lung epithelia, and especially in lung adenocarcinoma higher levels of the molecule were associated with better prognosis, acting as suppressor gene [12]. Because furin protein is a calciumdependent serine endoprotease, intracellular interactions with regucalcin remain unexplored in SARS-CoV-2 -mediated COVID-19. In contrast, regucalcin is involved in other viral infections -mainly in hepatitis B virus (HBV) - and its expression is correlated to the severity of liver lesions [13].

Based on these critical clinic-pathological and genetic data, we observe that specific chromosome $\mathrm{X} p$ arm rearrangements or SNPs that affect predominantly hACE2 - but also TLR7/8, FOXP33, and RGN- genes could potentially be useful molecular biomarkers for explaining differences in infected male and female patients regarding the SARS-CoV-2 - related lung pathology lesions (severe acute interstitial pneumonia), response to specific anti-viral treatment, and also levels of infectivity (transmission dynamics)/mortality rates in two sexes (Fig. 1).

\section{Compliance with Ethical Standards}

Conflict of Interest The authors declare that they have no conflict of interest.

\section{References}

1. Wu A, Peng Y, Huang B et al (2020) Genome Composition and Divergence of the Novel Coronavirus (2019-nCoV) Originating in China. Cell Host Microb 27(3):325-328
2. Lukassen S, Chua RL, Trefzer T et al (2020) SARS-CoV-2 Receptor ACE2 and TMPRSS2 Are Primarily Expressed in Bronchial Transient Secretory Cells. EMBO J e105114-118

3. Coutard B, Valle C, de Lamballerie X, Canard B, Seidah NG, Decroly E (2020) The spike glycoprotein of the new coronavirus 2019-nCoV contains a furin-like cleavage site absent in CoV of the same clade. Antiviral Res 176:104742-104747

4. Tsiambas E, Papanikolaou V, Chrysovergis A et al (2020) Coronavirus in hematologic malignancies: targeting molecules beyond the angiotensin-converting enzyme 2 (ACE2) wall. Pathol Oncol Res 1-3

5. Buja LM, Wolf DA, Zhao B et al (2020) The emerging spectrum of cardiopulmonary pathology of the coronavirus disease 2019 (COVID-19): Report of 3 autopsies from Houston, Texas, and review of autopsy findings from other United States cities. Cardiovasc Pathol 48:107233-107243

6. Lewnard JA, Liu VX, Jackson ML et al (2020) Incidence, clinical outcomes, and transmission dynamics of severe coronavirus disease 2019 in California and Washington: prospective cohort study. BMJ 369:m1923-1933

7. Yamada S, Utsunomiya M, Inoue $\mathrm{K}$ et al (2004) Genome-wide Scan for Japanese Familial Intracranial Aneurysms: Linkage to Several Chromosomal Regions. Circulation 110:3727-3733

8. Zhong J, Yan Z, Liu D et al (2006) Association of AngiotensinConverting Enzyme 2 Gene A/G Polymorphism and Elevated Blood Pressure in Chinese Patients With Metabolic Syndrome. J Lab Clin Med 147(2):91-95

9. Conti P, Younes A (2020) Coronavirus COV-19/SARS-CoV-2 Affects Women Less Than Men: Clinical Response to Viral Infection. J Biol Regul Homeost Agents 34(2)

10. Li G, He X, Zhang L et al (2020) Assessing ACE2 Expression Patterns in Lung Tissues in the Pathogenesis of COVID-19. J Autoimmun:102463-49

11. Sawalha AH, Zhao M, Coit P, Lu Q (2020) Epigenetic Dysregulation of ACE2 and Interferon-Regulated Genes Might Suggest Increased COVID-19 Susceptibility and Severity in Lupus Patients. Clin Immunol 215:108410-108415

12. Yamaguchi M, Osuka S, Shoji M, Weitzmann MN, Murata T (2017) Survival of lung cancer patients is prolonged with higher regucalcin gene expression: suppressed proliferation of lung adenocarcinoma A549 cells in vitro. Mol Cell Biochem 430(1-2):3746

13. Wei X, Yu H, Zhao P, Xie L, Li L, Zhang J (2019) Serum regucalcin is a useful indicator of liver injury severity in patients with hepatitis B virus-related liver diseases. Braz J Med Biol Res 52(10):e8845-e 8850

Publisher's Note Springer Nature remains neutral with regard to jurisdictional claims in published maps and institutional affiliations. 